Olivier Cousin

CADIS/CNRS/EHESS

Université Victor Segalen Bordeaux-2

3 ter, place de la victoire

33076 Bordeaux cedex

cousin@ehess.fr

\title{
La construction des inégalités hommes/femmes dans l'entreprise. Une analyse de cas dans la métallurgie*.
}

\section{Résumé}

Les lois sur l'égalité professionnelle, de 1983 puis de 2001, obligent les entreprises à présenter un rapport «sur la situation comparée des hommes et des femmes » au comité d'entreprise. Ce rapport sert de point d'appui aux négociations collectives et aux mesures correctives. Or, la comparaison n'est envisagée que dans l'instantanée, une année par rapport à la précédente, elle n'appréhende donc pas les différences de parcours et d'opportunités et risque ainsi d'occulter les inégalités entre les hommes et les femmes. A partir d'une étude de cas, réalisée dans une grande entreprise du secteur de la métallurgie, nous opposerons à ce rapport une analyse longitudinale et montrerons ainsi que les inégalités perdurent et se creusent parfois. Si l'entreprise étudiée se conforme à la loi, nous faisons cependant l'hypothèse que ce mode de présentation sert moins à corriger les inégalités qu'il ne lui permet de se protéger contre les plaintes et les procès pour discrimination.

Mots-clés : Inégalité hommes/femmes ; discrimination ; techniciens ; gestion des parcours

\begin{abstract}
The 1983 and 2001 laws on equality at work make it necessary for enterprises to produce a report « outlining a comparative assessment on the condition of men and women » on committee meetings. This report lays the groundwork for further collective negotiations and possible qualifying action to be taken. Now, the comparison only sets out to dwell on short-term durations extending over one year. As a result, it does not grasp various trajectories and scopes, and may well screen inequalities between men and women. Drawing on a case study conducted in a large metallurgy-related concern, we'll put forward a longitudinal survey against this report and show that inequalities are long-lived and sometimes increasing. However law-abiding the aforesaid concern may be, we suggest that this procedure is less an answer to inequality than a way to avoid prosecution.
\end{abstract}

Key words : Gender studies, Discrimination, Technicians, Life stories.

\footnotetext{
*. Je tiens particulièrement à remercier Georges Felouzis, Université Victor Segalen Bordeaux2, pour son aide et ses conseils sans lesquels les traitements des données n'auraient pu être mené à bien.
} 
Comment, dans le cadre d'une entreprise, appréhender les inégalités entre les hommes et les femmes ? Quels sont les critères retenus et que nous disent-ils de la manière dont l'entreprise s'empare de ces questions? Les indicateurs ne sont jamais neutres, même s'ils semblent s'imposer «naturellement » parce qu'ils sont ceux dont les entreprises disposent et sur lesquels elle peut rendre des comptes. Concernant les inégalités entre les hommes et les femmes, l'entreprise se soumet à une loi l'obligeant à fournir un « rapport annuel relatif à la situation comparée des hommes et des femmes » au comité d'établissement quand le nombre de salariés est supérieur à cinquante. Ce rapport détaille, selon des modalités précises, la situation des hommes et des femmes au regard de leur rémunération, de leur accès à la formation et de leur promotion... Il rend compte des égalités et des inégalités existantes et sert de point d'appui pour envisager des mesures correctives. Or, ces indicateurs introduisent un biais, principalement parce qu'ils abordent les données dans l'instantanée et ne tiennent pas compte des différences de parcours dans l'entreprise, ce faisant les inégalités n'apparaissent pas.

A partir d'une étude de cas, réalisée dans une très grande entreprise du secteur de la métallurgie, une lecture longitudinale des parcours des Techniciens sera entreprise et opposée au bilan produit par l'entreprise lors de la publication de son rapport. Alors que le rapport annuel laisse entrevoir une égalité de traitement entre les techniciens et les techniciennes, l'analyse détaillée de leur parcours montre, à l'inverse, que les inégalités perdurent, se creusent parfois, parce qu'à l'origine, lors de leur entrée dans l'entreprise, les unes et les autres sont dans des situations très inégales. Ainsi, le risque dans l'usage de ces indicateurs est d'occulter la réalité et finalement de maintenir les inégalités.

\section{UNE EGALITE APPARENTE}

Depuis la loi de 2001, venant compléter celle de 1983, les entreprises de plus de cinquante salariés doivent faire de l'égalité professionnelle un des objectifs majeurs de la négociation collective. L'égalité s'applique à tous les domaines des relations du travail: recrutement, rémunération, promotion et formation, et se traduit par des accords renégociables au bout de trois ans. L'application de la loi se matérialise par une présentation annuelle par l'employeur au comité d'entreprise d'un rapport sur la « situation comparée des hommes et des femmes ». Le rapport s'appuie sur des critères précis, définis par l'article D.432-1 du code du travail [A. Dauverchain, 2003 ; J. Laufer, 2005]. C'est à partir de ces 
indicateurs que des mesures peuvent être engagées afin de corriger les inégalités constatées. Les indicateurs sont simples et couvrent plusieurs domaines : la durée et l'organisation du travail ; la répartition selon les divers types de congés ; le nombre d'embauches et de départs ; le positionnement dans l'entreprise sur les grilles de classification ; le nombre de promotion ; la répartition selon les rémunérations ; l'accès à la formation et enfin, les conditions de travail (voir en annexe).

Au-delà des indicateurs, il est intéressant d'observer quel usage en fait l'entreprise et plus généralement quelle image elle construit d'elle-même. Nous ne retiendrons qu'un indicateur : la répartition des hommes et des femmes sur les grilles de classification définie par la branche de la métallurgie, branche à laquelle l'entreprise SimOUN, dont les données exploitées ci-après sont issues, appartient.

Le rapport, tel qu'il a été présenté au comité d'entreprise en 2003, compare la situation des femmes par rapport à celle des hommes au regard de l'année précédente. Il est soumis au comité d'entreprise, donc aux partenaires sociaux, et revêt à ce titre un caractère public. En revanche, il est établi par des membres de la direction du personnel et n'est pas construit collégialement. Il reflète ce que l'entreprise veut bien livrer et exposer à la critique. Or, si le choix d'une comparaison d'une année donnée par rapport à la précédente n'est pas en soi contestable, force est de constater qu'il introduit un biais important car jamais n'apparaît les situations de départs des hommes et des femmes. Certes, l'entreprise ne fait qu'appliquer la loi et utilise les indicateurs conformes aux obligations légales. Cependant, on peut faire l'hypothèse qu'à s'en tenir au cadre de la loi, l'entreprise cherche moins à affronter la question des inégalités hommes femmes qu'à éviter de s'engager dans un processus très lourd et sous la menace constante de plainte dirigée contre elle.

\section{Les grilles de classification}

L'étude de cas ne porte que sur une partie du personnel de cette très grande entreprise de 10400 salariés en octobre 2004 : les Techniciens ${ }^{1}$. Chez Simoun, ils sont le groupe le plus important, et ils sont au carrefour de deux types de mobilité : les ouvriers et les employés qui deviennent Techniciens, et les Techniciens qui deviennent cadres. L'étude de leurs

1. Par convention, et pour faciliter la lecture, dans la suite du texte l'usage de la majuscule désignera les Techniciens en tant que catégorie socio professionnelle, les minuscules serviront à distinguer les techniciennes des techniciens. 
parcours et de leurs perspectives de carrière permet donc d'appréhender sous différents angles la question des inégalités à partir d'une exploitation des fichiers du personnel.

L'entreprise appartient à la branche de la métallurgie. Des accords, de branche et d'entreprise, régissent la carrière des Techniciens. Ces accords distinguent deux grands types de populations: les cadres, qui ont leur propre grille; les ouvriers, les employés, les Techniciens et les agents de maîtrise qui se répartissent sur différents échelons. Chaque échelon est un coefficient multiplicateur de l'appointement minimum définit entre les partenaires sociaux. Quatorze coefficients, regroupés en cinq niveaux, classent les Techniciens : Niveau II (coefficient 170/180/190) ${ }^{2}$; Niveau III (coefficients 215/225/240); Niveau IV (coefficient 255/270/285); Niveau V (coefficient 305/335/365); Niveau VI (coefficient 395/425) ${ }^{3}$. Pour appréhender les inégalités, ce sont les échelons de départ et d'arrivée qui serviront de point de comparaison, et non pas le salaire car lorsque l'on travaille à partir des fichiers du personnel, le salaire n'est pas toujours accessible, ni fiable et, surtout, l'information est parfois incomplète puisque au salaire net il faut toujours ajouter un ensemble de primes qui n'apparaît que très rarement. Par ailleurs, le coefficient a une valeur symbolique. Il fonctionne comme l'indicateur de la place occupée par le salarié dans l'entreprise, il résume son degré de compétence, de savoir faire et de responsabilité dans son secteur d'activité. Grimper d'un échelon est systématiquement perçu comme une marque de reconnaissance plus valorisante que l'obtention d'une augmentation.

\section{Une égalité apparente}

Le rapport sur « la situation comparée des hommes et des femmes », publié en mai 2003 par SIMOUN pour les effectifs de 2002, met en avant une égalité de traitement, voire un léger avantage pour les femmes. En effet, toutes catégories professionnelles confondue, $13,19 \%$ des femmes ont eu une promotion entre 2001 et 2002, 13,48\% pour les hommes ; $16,3 \%$ des techniciennes, $14,1 \%$ pour les techniciens ${ }^{4}$. « Le nombre moyen d'heures d'action de formation »selon le sexe ne varie qu'à la marge : 37 heures pour les techniciennes ; 41

2. Dans la suite de la présentation de la répartition des Techniciens sur les coefficients, les Niveaux II et III seront regroupés car ils ne concernent qu'une minorité de ce groupe.

3. A titre d'exemple, l'appointement minimum en octobre 2004 a été fixé à 6,6073€ pour 36 heures travaillées, un Technicien situé au coefficient 190 gagnera au minimum $1255 €$ plus $22,93 €$ de part fixe, soit $1278,3 €$.

${ }^{4}$. Le rapport ne porte que sur les salariés d'un site qui accueille $40 \%$ de l'ensemble des effectifs. Cependant, à plusieurs reprises la commission indique que les données du site sont très proches de celle de l'ensemble de l'entreprise. Les informations mentionnées ne servant que d'illustration, on peut donc les généraliser à l'entreprise dans son ensemble. 
heures pour les hommes. Enfin, la comparaison des «éventails de rémunération »va dans le même sens pour les Techniciens à coefficient équivalent, comme le montre le tableau I cidessous.

Tableau I

Eventail des rémunérations mensuelles (en Euros) pour la population des Techniciens sur un des sites de l'entreprise en 2002.

\begin{tabular}{|c|c|c|c|c|c|c|c|c|c|}
\hline \multirow{3}{*}{$\begin{array}{c}\text { Coeff } \\
240\end{array}$} & \multicolumn{3}{|c|}{ Femmes } & \multicolumn{3}{|c|}{ Hommes } & \multicolumn{3}{|c|}{$\mathrm{F} / \mathrm{H}, \%$} \\
\hline & $\operatorname{mini}$ & moyen & $\max$ & $\operatorname{mini}$ & moyen & $\max$ & $\operatorname{mini}$ & moyen & $\max$ \\
\hline & 1784 & 1911 & 2100 & 1599 & 1972 & 2361 & 111,6 & 96,9 & 88,9 \\
\hline 255 & 1812 & 2011 & 2310 & 1791 & 2109 & 2525 & 101,2 & 95,4 & 91,5 \\
\hline 270 & 1938 & 2148 & 2473 & 1917 & 2217 & 2581 & 101,1 & 96,9 & 95,8 \\
\hline 285 & 2075 & 2279 & 2646 & 1844 & 2302 & 2843 & 112,5 & 99,0 & 93,1 \\
\hline 305 & 2150 & 2481 & 2874 & 2168 & 2489 & 3011 & 99,2 & 99,7 & 95,5 \\
\hline 335 & 2368 & 2633 & 2995 & 2375 & 2712 & 3229 & 99,7 & 97,1 & 92,8 \\
\hline 365 & 2658 & 2861 & 3314 & 2583 & 2938 & 3475 & 102,9 & 97,4 & 95,4 \\
\hline 395 & & & & 2879 & 3383 & 4163 & 0,0 & 0,0 & 0,0 \\
\hline 425 & & & & 3417 & 3760 & 4217 & 0,0 & 0,0 & 0,0 \\
\hline
\end{tabular}

Source : rapport sur la situation comparée des hommes et des femmes, 2002, mai 2003, Direction de l'établissement Pour chaque coefficient, trois catégories de salaires sont calculées rendant compte des augmentations individuelles.

Le tableau laisse entrevoir qu'au mieux les femmes gagnent, à coefficient équivalent, plus que les hommes (112\%), au pire $89 \%$ du salaire des hommes. L'entreprise apparaît comme exemplaire et se démarque des enquêtes qui, elles, soulignent les différences encore très importantes entre les hommes et les femmes ${ }^{5}$. Cependant, cette lecture est trompeuse. Le tableau compare la situation des hommes et des femmes, à coefficient équivalent, donc ici lorsqu'ils et elles occupent des postes similaires et ont le même type d'activité. Or, si encore trop souvent à travail égal le salaire, lui, demeure inégal, ces données ne disent rien des inégales possibilités pour les femmes d'atteindre le même coefficient que les hommes, donc finalement des probabilités parfois faibles d'assurer les mêmes types d'activité et d'occuper les mêmes postes. Le tableau I le suggère néanmoins puisque pour les coefficients les plus élevés (395 et 425) les données concernant les femmes ne sont pas fournies car elles ne sont pas significatives, et pour cause, elles sont très peu à atteindre ces seuils en 2003. Les données issues des fichiers du personnel font eux apparaître ces écarts : 2,2\% des femmes se situent aux coefficients $395 / 425 ; 5,6 \%$ pour les hommes. C'est sur ce registre que se joue l'essentiel des différences : l'inégalité d'accès aux différents coefficients.

\footnotetext{
5 Les femmes travaillant à temps complet gagnaient 66,7\% du salaire des hommes en 1970, 76,6\% au début des années 2000. [R. Silvera, 1998] ; INSEE, DADS 2003
} 


\section{UNE GESTION DISCRIMINANTE}

Sortir de cette illusion d'optique suppose d'appréhender les inégalités à partir d'une lecture permettant de comparer les parcours des Techniciens. Pour cela, deux périodes ont été retenues : leur situation en 1995 puis en 2003. Ces deux années seront observées en tant que telle, puis dans leur continuité à partir de la reconstruction de cohortes ${ }^{6}$ offrant la possibilité de reconstruire les parcours des Techniciens présents en 2003 et déjà salarié dans cette entreprise près de dix ans auparavant. Enfin, il a aussi été possible de suivre les salariés ayant changé de catégorie professionnelle : les ouvriers et les employés promus Techniciens, et les Techniciens qui deviennent cadres. Pour chaque moment, les données brutes seront exploitées ainsi que des analyses de régressions logistiques comprenant: le sexe, l'ancienneté dans l'entreprise, l'âge, le niveau de diplôme, la direction de rattachement et, mais uniquement pour l'année 2003, le type de métier. Seules les femmes travaillant à temps complet ont été retenues dans les régressions, afin de ne pas biaiser les observations ${ }^{7}$.

La discrimination sera ici abordée dans un sens économique, c'est-à-dire lorsque des individus avec les mêmes caractéristiques économiques reçoivent des salaires différents et que les différences sont systématiquement corrélées avec certaines caractéristiques nonéconomiques. Comme le suggère cette définition, la discrimination n'évoque pas seulement un phénomène négatif envers des individus appartenant à certains groupe, mais également un comportement de népotisme envers les membres de l'autre groupe, lui majoritaire ou dominant ${ }^{8}$.

En 1995, l'entreprise emploie 10185 salariés répartis sur plusieurs sites. Parmi ceuxci, les Techniciens représentent 43\% de l'effectif total. En 2003, l'effectif total est de 8247 salariés, dont $40 \%$ de Techniciens ${ }^{9}$. L'entreprise se caractérise par une population expérimentée qui y fait pour la très grande majorité toute sa carrière. Surtout, la population des Techniciens ne se renouvelle guère et c'est ainsi que 66\% d'entre eux ont plus de vingt

6. Il ne s'agit cependant que d'une reconstruction partielle puisque nous ne savons pas ce qu'on «fait» les salariés pendant cette période, notamment pour les femmes : congé de maternité, temps partiel...

7. Le travail à temps partiel concerne principalement les femmes. Il les pénalise parce qu'il représente un frein aux promotions, et aussi parce que le taux de rémunération horaire est plus faible. Le salaire horaire moyen du temps partiel s'élève à $71 \%$ du salaire horaire à temps plein. [R. Silvera, op. cit].

8. Définition proposée par J-E. Stiglitz, [J-E. Stiglitz, 1973], citée par O. Joseph, S. Lemière, [O. Joseph, S. Lemière, 2005]

9. Les deux années de référence prises en compte dans l'étude compte en réalité un plus grand nombre de salariés (11663 en 1995 et 10608 en 2003). Les effectifs ont été réduits car les salariés en arrêt pour longue maladie ou ceux en pré retraite n'ont pas été retenu. Par ailleurs, par souci d'harmonisation des fichiers nous n'avons gardé que les sites existant les deux années de référence. Les sites existant en 1995, mais fermés en 2003, ou ce qui sont entrés dans la société après 1995 n'apparaissent donc pas. 
ans d'ancienneté en 2003 (34\% en 1995). Elément qui aura une incidence importante sur les carrières.

Les techniciennes, elles, représentent $15 \%$ des effectifs, part qui n'a pratiquement pas évolué sur la période considérée ${ }^{10}$. En 1995, comme en 2003, elles ne se démarquent guère quant à leur ancienneté dans l'entreprise et leur moyenne d'âge ${ }^{11}$. Par ailleurs elles sont globalement plus diplômées que les hommes ${ }^{12}$. Elles possèdent donc a priori des atouts favorables au déroulement de leur carrière et, en toute logique, compte tenu du lien entre diplôme, ancienneté et promotion ${ }^{13}$, elles devraient être systématiquement mieux placées sur les grilles de classification ou, au pire, faire jeu égal. Or, il n'en est rien et elles ne bénéficient que marginalement de leur avantage.

\section{Les inégalités effectives}

Les hommes et les femmes n'ont pas les mêmes opportunités parce qu'au départ ils ne sont pas traités de la même manière et ne bénéficient pas de la même attention. Ainsi, une étude du CEREQ montre que dans les premières années de la vie active, le salaire moyen des hommes croît beaucoup plus que celui des femmes [A. Dupray, S. Moullet, 2005] ${ }^{14}$. Ce handicap pèsera tout au long de la carrière des femmes. C'est cet élément qu'il convient d'introduire et qui n'est pas pris en compte par le rapport annuel sur la situation comparée des hommes et des femmes.

10. En 1995, les femmes représentent 14\% des Techniciens, 15\% en 2003. Dans l'ensemble de l'entreprise, elles comptent pour $14 \%$ des salariés. Il n'y a que parmi la population des cadres que leur nombre a sensiblement augmenté : 14\%; $18 \%$.

11. En 1995, en moyenne, les femmes ont 41 ans, idem pour les hommes et 16 ans d'ancienneté, 17 ans pour les hommes. En 2003, elles ont 46 ans, idem pour les hommes, et 22 ans de présence, 23 pour les hommes.

12. En 1995: 46\% des femmes ont un CAP/BEP, $20 \%$ un bac, $34 \%$ un bac+2 et plus; $45 \%, 26 \%, 29 \%$ respectivement pour les hommes. En 2003: 40\% CAP/BEP, 21\% bac, 38\% bac +2 et plus pour les femmes ; $44 \%, 26 \%, 30 \%$ respectivement pour les hommes. Il est à noter que concernant le diplôme, les effectifs de référence sont moindre car certaines données sont erronées, elles ont été éliminées.

13. L'ancienneté est une variable plus discriminante que l'âge, qui lui est par ailleurs fortement corrélé compte tenu de la très grande stabilité des salariés dans l'entreprise. Le coefficient de détermination explique $12 \%$ de la variance pour l'ancienneté et $15 \%$ pour le diplôme.

14. Dans les toutes premières années de la vie active, dans le secteur privé pour les salariés à temps plein, le salaire des hommes croît en moyenne de 7,5\% par an ; 6,5\% pour les femmes. 
Tableau II

Chances d'accéder aux coefficients les plus élevés pour les Techniciens en 1995

Régression logistique

\begin{tabular}{|l|l|c|c|c|}
\hline Variable & Modalité & B & Sig & Odds Ratio \\
\hline Constante & & $-5,61$ & 0,000 & \\
Femme & & & & \\
\hline Ancienneté & Homme & 1,15 & 0,000 & 3,18 \\
\hline Age & & 0,11 & 0,000 & 1,11 \\
\hline Diplôme & & 0,06 & 0,000 & 1,06 \\
Bac +4 & CAP & $-2,06$ & 0,000 & 0,12 \\
& Bac & $-0,96$ & 0,000 & 0,38 \\
& Bac+2 & 0,45 & N.S & \\
\hline Directions & & & & \\
Technique & Autres & $-0,007$ & N.S & \\
& Civile & $-0,37$ & N.S & \\
& Militaire & 0,29 & N.S & \\
& Production & $-0,31$ & 0,007 & 0,7 \\
& Qualité & $-0,14$ & N.S & \\
\hline
\end{tabular}

Variable à tester : probabilité d'atteindre le coefficient 335 ou plus pour les techniciens. Variable discontinue $0=<$ $335 ; 1=>335$

Lecture : L'individu de référence est une femme, moins de 3 ans d'ancienneté, bac+4, direction technique. Les hommes, toutes choses égales par ailleurs, ont 3 fois plus de chance d'être au coefficient 335.

Toutes choses égales par ailleurs, être une femme représente un handicap pour atteindre le coefficient 335 et plus, soit de faire partie de la catégorie des $20 \%$ les mieux placés sur l'échelle de classification. Les hommes ont 3 fois plus de chance que les femmes d'atteindre ce niveau. Dans la répartition des hommes et des femmes sur les coefficients, en 1995, 26,5\% des premiers sont au coefficient 335 ou au-delà, alors que ce n'est le cas que pour $10,5 \%$ des techniciennes. Quand le sexe et l'ancienneté sont associé dans le modèle (voir tableau VIII en annexe) la nature des inégalités est encore plus apparente. L’interaction entre le sexe et l'ancienneté est significative et positive, ce qui signifie qu'elle joue en la faveur des hommes. Pour les femmes, la situation est différente puisque l'interaction n'est pas significative tant qu'elles n'entrent pas dans la dernière partie de leur carrière (20 ans et plus d'ancienneté). Cependant, même à partir de ce seuil, l'interaction leur est moins favorable. En effet, les femmes ayant entre 20 et 40 ans d'ancienneté dans l'entreprise ont 1,9 fois plus de chance que les hommes débutants d'atteindre le coefficient 335 et plus, opportunité nettement moindre que pour les hommes qui sont dans la première partie de leur carrière (entre 11 et 19 ans). Dans l'ensemble donc une année supplémentaire d'expérience professionnelle a un rendement salariale plus faible pour les femmes que pour les hommes [A-M. Daune Richard, 1998]. Il faut, à titre d'exemple, sept ans d'ancienneté aux hommes pour que $10 \%$ d'entre eux soit au coefficient 335 et plus. Il en faut dix-neuf aux femmes.

En 2003, presque dix ans plus tard, les écarts persistent, mais s'atténuent sensiblement. Les variables introduites dans le modèle restent significatives, mais leur poids 
est moindre. Les hommes ont, toutes choses égales par ailleurs, 2,6 fois plus de chances d'être au coefficient 36515 et plus que les femmes (voir en annexe, tableau VII et IX).

Les inégalités hommes/femmes ne se réduisent pas seulement à un moindre rendement de l'expérience professionnelle pour les techniciennes. Le diplôme joue aussi un rôle déterminant et globalement, quel que soit le secteur d'activité, plus il est élevé plus les salariés ont des opportunités d'atteindre les plus hautes marches des entreprises. Or, dans le cas présent, il joue un rôle complexe et ambigu. En effet, les techniciennes sont dans l'ensemble plus diplômées que les hommes, aussi bien en 1995 qu'en 2003, et pourtant elles n'en tirent que marginalement profit. A diplôme équivalent, elles n'ont pas les mêmes opportunités. Par exemple, $58 \%$ des hommes titulaires d'un CAP se situent au Niveau V (coefficient 305/335/365), alors que seulement 29\% des femmes y accèdent. Il n'y a que lorsqu'elles possèdent un bac +2 qu'elles font jeu égal avec les hommes, mais dans ce cas toutefois, ces derniers atteignent plus souvent le Niveau VI. En 2003, 10\% des hommes avec un bac +2 sont à ce niveau (coefficient 395/425) quand seulement 4\% des femmes y arrivent. Le déclassement des diplômes est donc bien plus accusé pour les femmes que pour les hommes [C. Marry, 1997]. Dans le cas des techniciennes, le diplôme n'agit pas pleinement car, si tel était le cas, elles devraient être systématiquement positionnées sur les coefficients les plus élevés. En même temps, il amortit partiellement la discrimination, car s'il y avait égalité entre les hommes et les femmes quant à leur niveau scolaire, donc si elles aussi étaient majoritairement peu diplômées, alors la discrimination serait encore plus importante, en particulier pour celles qui ont un CAP/BEP et un Bac.

\section{L’illusion de la réduction des écarts}

Ce que le «rapport sur la situation comparée des hommes et des femmes » laisse d'abord entrevoir c'est que les femmes participent bien d'un mouvement général où chaque année dans cette grande entreprise au moins $15 \%$ des salariés bénéficient d'une promotion individuelle. Elles ne sont donc aucunement exclue d'un vaste mouvement comparable à un escalier mécanique. Chaque salarié y est embarqué et, sur une période de près de dix ans, pratiquement tous les Techniciens ont eu au moins une promotion. Il existe donc une sorte d'aspiration générale tirant les Techniciens vers le haut. Entre 1995 et 2003, les plus faibles

\footnotetext{
15. Pour chaque période considérée, la régression logistique teste, pour un individu donné, la probabilité de faire partie des $20 \%$ des salariés situés sur les plus hauts coefficients. Compte tenu de l'augmentation moyenne de l'ancienneté des Techniciens, le seuil en 2003 n'est plus au coefficient 335, mais à l'échelon immédiatement supérieur : le coefficient 365.
} 
coefficients (de 190 à 240) tendent à disparaître et près des deux tiers des Techniciens (61\%) se situent au Niveau V (coefficients 305/335/365) en 2003. Les femmes suivent le mouvement et désormais près de la moitié d'entre elles atteignent ce Niveau (27\% en 1995). La reconstruction des cohortes l'atteste. Sur 3294 Techniciens salariés en 2003, nous disposons d'informations fiables pour 2630 qui étaient déjà présents en 1995. Il est donc possible de construire des tables de mobilité (voir tableaux X et XI en annexe) et de comparer les situations de départ avec celles d'arrivée. Seul 18\% d'entre eux n'ont connu aucune promotion et les taux sont strictement équivalents pour les hommes et pour les femmes. Il ne semble donc pas y avoir de différence.

Toutefois, cette lecture n'est que partiellement vraie car elle confond inégalité et exclusion, et c'est en partie celle qui est suggérée dans le rapport sur la situation comparée publié par l'entreprise. Il s'agit ici de distinguer l'égalité d'accès à un bien, ici la possibilité d'avoir une promotion, et l'égalité d'accès à des biens de qualités égales, ici les plus hautes marches, pour reprendre la distinction de M. Duru-Bellat à propos des inégalités à l'école [M. Duru-Bellat, 2002].

C'est cette question qui se pose à propos de la réduction des inégalités. Dans cette entreprise, au fil du temps, les écarts entre les techniciennes et les techniciens, quant à leurs opportunités de carrière, s'aggravent-ils, se réduisent-ils ou se maintiennent-ils? L'observation de la cohorte entre 1995 et 2003 apporte deux types de réponse. Globalement, il y a bien égalité d'accès à un bien : connaître au moins une promotion sur cette période. Quel que soit le coefficient de départ en 1995 et le sexe, 82\% des Techniciens bénéficient d'une promotion. En revanche, lorsqu'il s'agit d'apprécier qu'elles sont les opportunités de connaître au moins deux promotions durant cette période, les inégalités perdurent et s’aggravent parfois selon les points de départs, comme le montre le graphique I. 
en fonction de leur coefficient de départ en 1995.

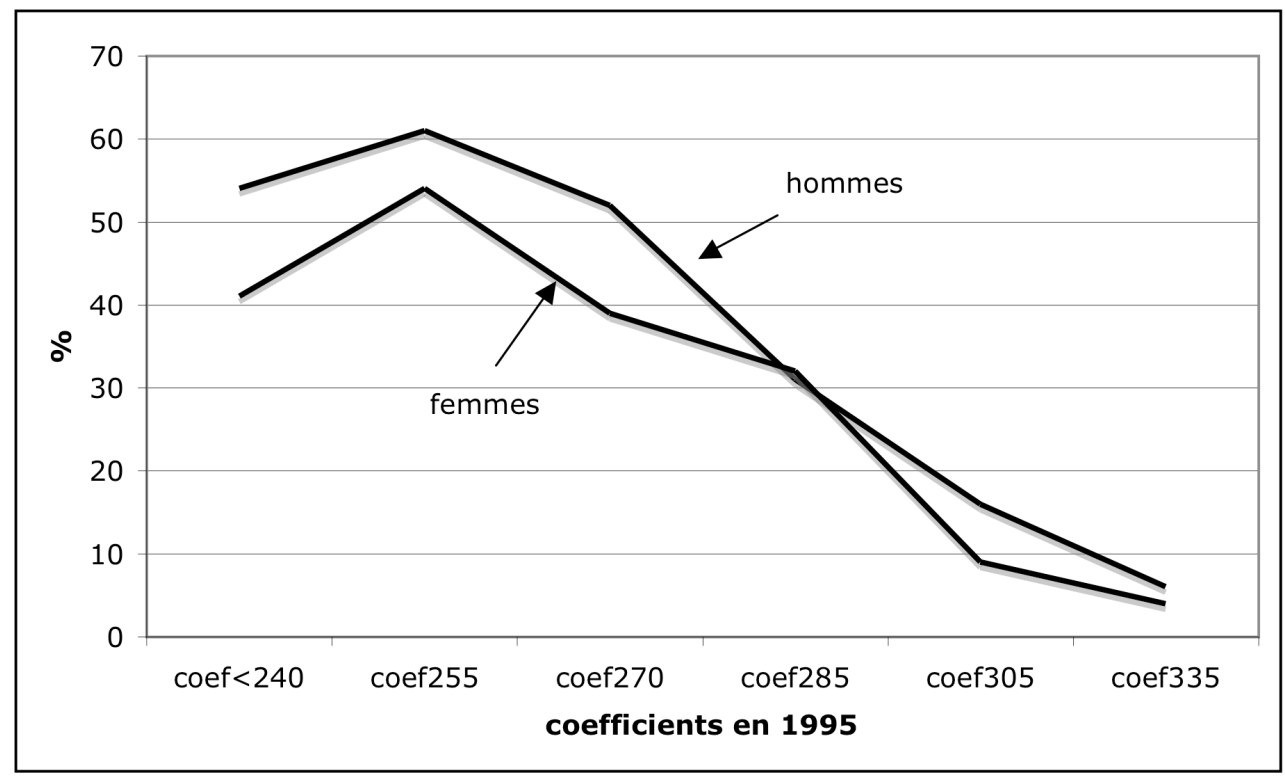

Le graphique est construit à partir des tables de mobilité présentée en annexe.

A l'exception notable des Techniciens situés sur le coefficient 285 en 1995 où les hommes et les femmes ont pratiquement les mêmes chances d'avoir deux promotions en près de dix ans, dans tous les autres cas, les opportunités des techniciennes sont moindres. C'est dans les plus bas échelons en 1995 qu'on leur offre le moins souvent la possibilité d'avoir deux promotions : $41 \%$ des femmes situées sur un coefficient inférieur ou égal à 240 ont cette opportunité, contre $54 \%$ des hommes. Or, d'une part, ce sont à ces échelons que les Techniciens ont le plus de chances de gravir des marches, puisque par définition l'éventail est le plus grand. D'autre part, les femmes, en 1995, sont beaucoup plus souvent à ce palier que les hommes (20\% contre $7 \%$ ). Elles ont donc au final beaucoup moins d'opportunités que les hommes. Toujours dans cette perspective, quelques hommes au coefficient 240 ont la possibilité d'accéder à l'échelon 305, donc de grimper quatre marches et d'être ainsi situés au Niveau V, soit le palier dans le lequel se retrouve la très grande majorité des Techniciens en 2003, alors qu'aucune femme n'y parvient.

L'analyse des variations en fonction de l'âge permet de saisir en partie comment s'opère la discrimination à l'égard des femmes qui, si elle ne relève pas d'une pratique volontaire, s'appuie sur les stéréotypes de sexe quant à la disponibilité des femmes et à leur capacité d'avoir de plus grandes responsabilités. En effet, en scindant la population issue de la reconstruction de la cohorte en deux, en dissociant d'un côté les Techniciens ayant moins de trente-neuf ans et de l'autre ceux qui ont plus de quarante ans, on s'aperçoit que les opportunités de connaître au moins deux promotions entre 1995 et 2003 varient fortement. Ce 
sont les jeunes femmes (- de 39 ans) qui sont le plus durement sanctionnées, c'est-à-dire lorsqu'elles sont susceptibles d'attendre des enfants et quand ces derniers sont encore très jeunes et nécessitent une plus grande présence. Quelle que soit leur situation réelle, cette période agit comme un stigmate alors même que dans l'échantillon seules les femmes travaillant à plein temps ont été retenues. Ce qui entrave leur mobilité, durant cette période, ce sont soit les congés de maternité pris lors des naissances, soit les préjugés concernant une disponibilité qui ne peut être que moindre compte tenu de la division sexuelle du travail domestique. Or, après quarante ans, comme le montre le graphique suivant, les femmes sont moins pénalisées et bénéficient même d'un léger avantage, cependant celui-ci est trop faible pour compenser les inégalités antérieures.

Graphique II, Cohorte 1995/2003

$\%$ des hommes et des femmes ayant gravi au moins deux échelons en fonction de leur coefficient de départ en 1995.

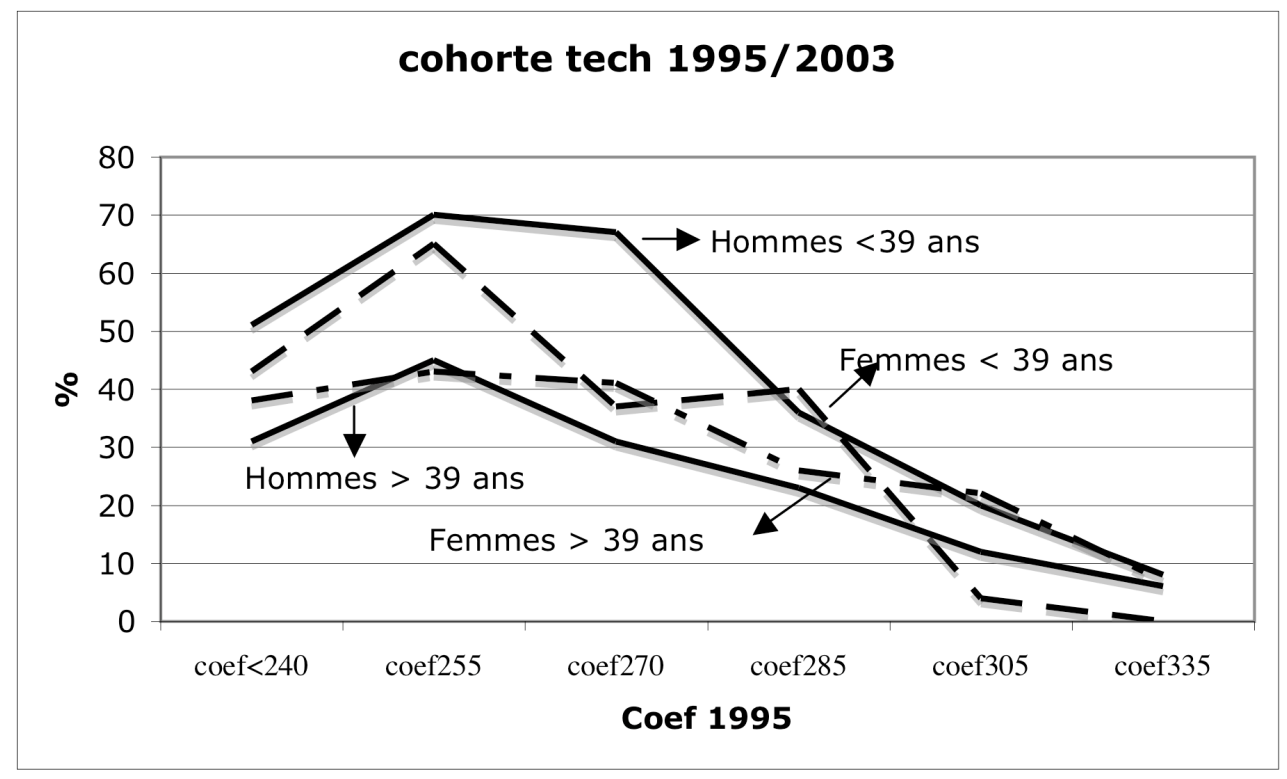

Les inégalités se redoublent donc. Les femmes partent avec un fort handicap, puisqu'elles sont principalement cantonnées sur les marches les plus basses. Puis on leur propose moins souvent qu'aux hommes d'avoir deux promotions sur une période de près de dix ans. L'égalité d'accès à un bien ne doit donc pas occulter les inégalités de départ qui aboutissent à ce que les femmes n'auront au final que peu de chances d'obtenir un bien de qualité égale. Car, comme le suggèrent les études de l'INSEE et du CEREQ, prenant en compte les situations des hommes et des femmes à emploi comparable en début de carrière, les écarts de salaire au départ perdurent et se creusent par la suite [S. Le Minez, S. Roux, 
2001 ; A. Dupray, S. Moullet, 2005]. L'égalité formelle, telle qu'elle apparaît a priori, tend à reproduire les inégalités de départ. Pour qu'elles puissent être corrigée, encore faut-il qu'elles apparaissent.

\section{Le poids des filières professionnelles}

Si les femmes partent avec un handicap qu'elles ne surmontent que marginalement c'est aussi parce qu'elles travaillent dans des filières professionnelles les moins valorisées. Que ce soit à l'école, puis après sur le marché du travail, le prestige des filières pèse lourdement sur les carrières. Les orientations scolaires qu'elles choisissent et qu'elles subissent les desservent et les écartent du champ de la technique, domaine qui ouvre le plus de perspective sur le plan des responsabilités en entreprise notamment [M. Duru-Bellat, 1990 ; C. Marry, 2004]. Dans la division du travail, les femmes en général occupent plus souvent les postes les moins prestigieux, ceux qui sont à la fois les moins payés et qui offrent le moins de perspectives [A-M. Daune Richard, 1998 ; S. Fortino, 2004]. Elles travaillent aussi plus fréquemment dans les secteurs les moins rémunérateurs : tertiaire, fonction publique.

Dans l'entreprise, dont sont extraites les données exploitées, ces éléments deviennent un facteur aggravant. SIMOUN se distingue en effet par la conception et la fabrication de produits à haute valeur ajoutée nécessitant une technologie de pointe. La technologie constitue le cœur et l'identité de l'entreprise, sa fierté. Or, sans grande surprise, les femmes en sont relativement écartées. Soixante-cinq types de métiers sont recensés, regroupés en dixsept catégories de famille de métiers ${ }^{16}$. Si les femmes sont minoritaires, elles se répartissent aussi inégalement sur les différentes catégories de métier. On peut ainsi distinguer deux grands groupes. Le premier accueille moins de $20 \%$ de femmes, par convention il sera appelé «métier non-féminin», dans le deuxième au contraire elles sont statistiquement surreprésentées, cet ensemble de métier sera appelé «métier féminin », même si elles restent minoritaires. Chez Simoun, les «métiers féminins », pour les Techniciens, sont au nombre de sept : Achat, Droit, Documentation, Ressources humaines, Logistique, Secrétariat et Vente. L'intitulé même de ces catégories de métier ne laisse aucune ambiguïté, ils sont considérés comme n'étant pas «spécifiques », c'est-à-dire, comme ne participant pas directement à la

\footnotetext{
16. Famille de métier par ordre d'importance décroissante : Qualité, Recherche et développement, Méthodes, Droit économie finances, Gestion de production, Maintenance moyen généraux, Logistique, Fabrication montage, Informatique, Essai, Management, Achat, Support client, Ressources humaines, Documentation, Secrétariat, Vente.
} 
production et à la fabrication du produit. Pour reprendre le vocabulaire d'usage, ils ne sont pas dans le «cœur de métier». Exercer ce type d'activité, c'est voir s'éloigner les perspectives d'accéder aux plus hautes marches.

Tableau III

Chances d'accéder aux coefficients les plus élevés pour les Techniciens en 2003

Régression logistique

\begin{tabular}{|l|l|c|c|c|}
\hline Variable & \multicolumn{1}{|c|}{ Modalité } & B & Sig & $\begin{array}{c}\text { Odds } \\
\text { Ratio }\end{array}$ \\
\hline Constante & & $-8,61$ & 0,000 & \\
\hline $\begin{array}{c}\text { Sexe } \\
\text { Femme }\end{array}$ & Homme & 0,78 & 0,000 & 2,2 \\
\hline Ancienneté & & 0,09 & 0,000 & 1,1 \\
\hline Age & & 0,1 & 0,000 & 1,11 \\
\hline Diplôme & CAP & $-1,98$ & 0,000 & 0,13 \\
Bac +4 & Bac & $-0,88$ & 0,004 & 0,41 \\
& Bac+2 & 0,48 & N.S & \\
\hline Directions & Autres & $-0,04$ & N.S & \\
Technique & Qualité & $-0,27$ & N.S & 0,57 \\
& Production & $-0,55$ & 0,000 & 0,22 \\
& Civile & $-1,51$ & 0,000 & N.S \\
\hline Métiers & Militaire & 0,30 & 0,003 & 1,56 \\
\hline "Féminin" & "Non féminin" & 0,44 & & \\
& & & & \\
\hline
\end{tabular}

$335 ; 1=>365$

Variable à tester : probabilité d'atteindre le coefficient 365 ou plus pour les techniciens. Variable discontinue $0=<$

Lecture : L'individu de référence est une femme, avec un an d'ancienneté, bac +4 et plus, direction technique et exerçant un «métier féminin ». Les hommes ont 2,2 fois plus de chance d'atteindre le coefficient 365 et plus.

Considérer la «nature » du métier exercé, n'empêche qu'être une femme est en soi pénalisant. Toutes choses égales par ailleurs, les hommes ont 2,2 fois plus de chances d'atteindre le coefficient 365 que les femmes. Cette opportunité est même légèrement supérieure que précédemment, quand le métier n'était pas introduit dans le modèle. Par ailleurs, le type de filière dans lequel elles s'engagent, ou dans lequel on les maintient, aggrave les différences. Exercer un métier «non féminin » offre 1,5 fois plus de chances d'atteindre les plus hauts coefficients, toutes choses égales par ailleurs. Une sorte de hiérarchie s'instaure donc, les femmes exerçant un « «métier féminin » sont celles qui ont le moins de chance d'être situées au coefficient 365 et plus, puis viennent les femmes ayant un métier «non féminin», puis les hommes pratiquent un «métier féminin», et enfin, les hommes exerçant un métier «non féminin ». Quand le modèle teste l'interaction entre le sexe et l'ancienneté, les ordres de grandeur reste les mêmes et les femmes qui ont plus de vingtcinq d'ancienneté ont certes plus de chances de faire partie des salariés les mieux payés que 
les hommes jeunes, toutes choses égales par ailleurs, mais cette opportunité est toujours moindre que pour leurs homologues masculins (voir tableau XII en annexe). En fin de compte, le «féminin », comme attribut, est doublement pénalisant. Les écarts constatés reflètent deux phénomènes : «le fait que les femmes, à compétence égales, peuvent être positionnées dans des postes hiérarchiquement inférieurs par rapport à leurs homologues masculins et le fait que les postes à dominante féminine peuvent être moins valorisées que celles des hommes à travers les coefficients hiérarchiques » [F. Fakhfkh, S. Lemière, M-P. Merlateau, D. Meurs, 2004].

Pour les hommes, exercer un «métier féminin » équivaut à perdre une part de leur avantage de départ. Ils auront un déroulement de carrière moins rapide, comme si pratiquer ce type de «métier » était dévalorisant. Pour les femmes, en revanche, exercer un métier «nonféminin » ne procure qu'un faible bénéfice dans les opportunités d'accéder au coefficient 365 . Les carrières des techniciennes sont assez proches quel que soit le type de métier pratiqué. La mixité, qui ici signifie pratiquer un métier «non féminin », offre des avantages et ouvre de plus grandes perspectives aux femmes, sans pénaliser les hommes faut-il le rappeler, mais ne remplit pas totalement ses promesses. En particulier parce qu'elle ne permet pas suffisamment de compenser le poids des déterminismes de genre. C'est ainsi que s'il faut 27 ans d'ancienneté aux hommes qui exercent des «métiers non féminins » pour que 5\% d'entre eux atteignent le coefficient 365 et plus, il faut 30 ans pour les hommes ayant un «métier féminin », 37 ans pour les femmes pratiquant des métiers «non féminins » et 40 ans, enfin, pour celles qui ne sont pas dans les «cœurs de métiers»17. Les écarts restent donc considérables. D'autant plus que la répartition des unes et des autres sur ces deux groupes de métier ne se fait pas au hasard. La mixité risque en réalité de ne corriger qu'à la marge les inégalités globales puisque $58 \%$ des femmes exercent un «métier féminin » contre seulement $15 \%$ des hommes. Dit autrement, $42 \%$ seulement des techniciennes bénéficient du petit avantage de se retrouver dans un univers « masculin ».

Le type de métier exercé relève des « choix » et des orientations scolaires faites par les filles tout au long de leur scolarité. Cependant, l'entreprise joue aussi un rôle en permettant ou en refusant l'accès au métier «non féminin» aux femmes dans le déroulement de leur carrière. Si les données accessibles n'offrent aucune indication en la matière, les propos recueillis auprès de quelques salariés montrent la résistance de l'entreprise à favoriser les

17. Ces données résultent d'un calcul probabiliste (gain marginal) permettant d'illustrer les résultats, cf. L-A Vallet, J-P Caille [L-A. Vallet, J-P. Caille, 1996, p. 50]. 
passerelles. «Moi, raconte une experte en ressource humaine présente depuis 25 ans dans l'entreprise, je suis arrivée comme technicienne dans un métier qui s'appelait technicien de sous-traitance. Moi, je voulais être technicien méthode. Je me suis fait casser. On m'a dit: “quand une femme passe à l'atelier, les hommes s'arrêtent de travailler". C'est peut-être vrai une fois, mais au bout d'un mois, c'est fini. Mais on n'a pas voulu, les postes des méthodes étaient fermés aux femmes. La discrimination, je le ressens surtout dans les a priori pour tenir tel ou tel poste. On ne m'a pas accordé la confiance et on préfère ne pas prendre de risques. Ce sont des postes à risques, donc les plus valorisés. Si les femmes n'y sont pas, elles ne sont pas sur la chaîne de valeur, celle qui permet d'avoir de l'ascension. » ${ }^{18}$ Une autre responsable en ressources humaines, présentes depuis 13 ans dans l'entreprise explique : «Le fait d'être une femme, sur la carrière, ça n'aide pas. On doit travailler trois fois plus. A "la fabrication", par exemple, il y a deux niveaux de conseiller ressources humaines. Les deux hommes en ont le chapeautage. Ici, il y avait un poste phare, c'est toujours un homme qui l'a tenu. Il a la gestion d'une grosse unité. C'est un poste sensible, il a la gestion de la population ouvrière, la gestion des heures sup. C'est un poste de management, il a deux personnes en dessous. Je ne crois pas que ce poste sera donné à une femme. »

Les techniciennes sont désavantagées en tant que femme car, quel que soit le modèle, la variable sexe est toujours très significative et le fait d'être un homme multiplie toujours les chances d'atteindre les plus hauts coefficient, toutes choses égales par ailleurs, et parce qu'elle sont en grande partie écartées des métiers techniques, ceux là même qui ouvrent les plus grandes opportunités. Ainsi, la discrimination à l'égard des femmes perdure et rien n'indique qu'elle s'atténue réellement en 2003 par rapport à 1995. Les femmes partent avec un handicap, qu'elles ne surmontent qu'à de très rares exceptions. Pendant leur carrière, les écarts risquent non pas de se stabiliser ou de se maintenir mais de se creuser parce qu'ils sont le résultat d'accumulation d'événements qui viennent les pénaliser. Il est plausible de penser que chaque année passée à exercer un «métier féminin » renforce les écarts puisque c'est chaque année une opportunité de moins de pouvoir obtenir une promotion quand on se retrouve dans un secteur jugé peu ou pas stratégique ${ }^{19}$. Elles payent là leurs « choix » et leurs orientations scolaires qui les conduisent, et parfois les enferment, dans les métiers non

\footnotetext{
18. Les citations sont extraites d'entretiens menés auprès de différents responsables de la direction des ressources humaines, dans le cadre d'une enquête plus large, sur le thème du travail, conduite dans cette entreprise entre 2003 et 2005 .

19. La non-mixité des emplois reste la variable principale expliquant la différence de salaire, note R. Silvera, [R. Silvera, 1998].
} 
techniques, puis par la suite entravent la mixité professionnelle [J. Laufer, 2003]. Comme le suggère C. Marry, à propos des femmes ingénieurs, la transgression, c'est-à-dire le dépassement des préjugés et le choix pour les filles d'opter pour les filières techniques, est payante, à condition cependant que les entreprises le permettent et l'encouragent dans leurs parcours professionnels 20 .

\section{INEGALITE FACE AUX MOBILITE INTER CATEGORIELLES}

«L'origine » des inégalités ne tient pas seulement aux types de métier exercé. Elle s'enracine aussi dans les parcours initiaux des Techniciens qui stigmatisent et hiérarchisent fortement l'ensemble des salariés, au point qu'ils ne parviennent jamais véritablement à s'en défaire alors même qu'ils ont connu diverses formes de mobilité. Le poids du passé se révèle être un élément important dans la carrière des salariés et pénalise beaucoup plus les femmes que les hommes. L'observation des mobilités inter catégorielles en offre un bon aperçu. En effet, les Techniciens sont au carrefour de deux types de parcours : une partie d'entre eux étaient auparavant ouvriers ou employés, d'autres vont devenir cadres. Dans les deux cas, il s'agit bien plus que d'une promotion. Symboliquement et statutairement, les premiers quittent l'univers des milieux populaires pour appartenir aux catégories intermédiaires, les seconds sont identifiés désormais comme faisant partie des cadres et professions intellectuels supérieurs, selon la nomenclature de l'INSEE. Si le passage n'est pas nécessairement ritualisé, au moins pour ce qui concerne l'accès au statut de Technicien, il n'en reste pas moins marquant dans la carrière des salariés, leur ouvrant d'autres fonctions, responsabilités et possibilité d'avancement.

L'entreprise offre un double visage. Le système est relativement ouvert puisque cette mobilité demeure une réalité alimentant ce qu'il est convenu d'appeler, dans l'univers des cadres, les «cadres autodidactes ». Toutefois, cette ouverture cache, une fois la mobilité assurée, des disparités importantes entre ceux qui sont Techniciens, ou cadres, à la sortie du système scolaire, et ceux qui y entrent par la «petite porte » ${ }^{21}$. De nouveau, l'accès à un bien ne saurait être confondu avec l'accès à un bien de qualité égale.

20. C. Marry, 2004, op. cit. Sur ce thème voire aussi M. Duru-Bellat, 1990, op. cit, qui émet plus de réserves.

21. Pour reprendre la distinction de P. Bourdieu, [P. Bourdieu, 1991]. 


\section{Devenir technicien}

Par rapport à l'effectif de 2003 (3933 Techniciens) 22, $36 \%$ n'étaient pas initialement Techniciens : 17\% sont d'anciens ouvriers (674; $47 \%$ de l'ensemble des promus), 13\% d'anciens employés $(515 ; 36 \%)$ et $6 \%$ sont d'anciens agents de mâtrise $(237 ; 17 \%)^{23}$. C'est dans la première partie de leur carrière que les promotions se font et, au regard de l'ancienneté moyenne dans l'entreprise pour les Techniciens (21 ans), l'accès à cette catégorie se fait assez tôt. Le changement de statut est à comprendre comme un pari sur l'avenir, une valorisation de compétence et de potentiel et non pas comme une récompense ultime [P. Veneau, 1995].

Pour les femmes, leur parcours est paradoxal. Elles représentent $24.5 \%$ des promues. Elles sont donc sur-représentées. Elles proviennent essentiellement de la catégorie des employées, seul groupe socioprofessionnel où elles sont en supériorité numérique. A elles seules, les employées représentent $91 \%$ des futures techniciennes. Surtout, la part des promues chez les techniciennes est étonnamment disproportionnée. Sur 100 techniciennes, 48 sont des promues ; 26 seulement pour les hommes ${ }^{24}$. Près de la moitié de la population des techniciennes est donc entrée dans l'entreprise en étant d'abord employée. Statut pénalisant au démarrage de leur carrière, puisque, par exemple, alors qu'elles sont sensiblement plus diplômées que les hommes, elles n'en sont pas moins situées sur les plus bas échelons au moment de leur passage (52\% d'entre elles sont au Niveau II et III, inférieur au coefficient $255 ; 44 \%$ pour les hommes). Au regard des conditions de passage dans la catégorie des Techniciens, le système est donc a priori peu discriminant. En revanche, il l'est quant aux opportunités d'évolution après ce passage. Les femmes se trouvent de nouveau pénalisées. Etre une femme, toutes choses égales par ailleurs (ancienneté et coefficient au moment du passage, catégorie d'appartenance, diplôme) divise par 2 les chances d'atteindre le coefficient 335 pour les promues par rapport aux hommes.

\footnotetext{
22. Concernant la mobilité inter catégorielle, le fichier de référence n'est plus le même et il prend en compte l'ensemble des salariés de l'entreprise en 2003, hors ceux en arrêts maladie et les femmes travaillant à temps partiel.

23. Les agents de maîtrise ne seront pas retenus en tant que tel, parce qu'il n'est pas certain qu'ils s'agissent à proprement parlé d'une promotion sociale et parce qu'il y a autant d'agents de maîtrise qui deviennent Techniciens (237), que de Techniciens qui passent agents de maîtrise (250).

24. Les données ici se démarquent de la situation moyenne que connaissent en général les femmes sur le marché du travail puisqu'elles connaissent une moindre mobilité professionnelle : $30 \%$ des hommes et 17\% des femmes employées accèdent au statut de profession intermédiaire. A-M ; Daune-Richard, 1998, op. cit.
} 
Tableau IV

Chances d'accéder aux coefficients les plus élevés pour les ouvriers et les employés devenus Techniciens

Régression logistique

\begin{tabular}{|l|l|c|c|c|}
\hline Variable & Modalité & B & Sig & Odds Ratio \\
\hline Constante & & $-24,76$ & 0,000 & \\
Homme & Femme & $-0,75$ & 0,01 & 0,48 \\
\hline Ancienneté & & $-0,09$ & 0,000 & 0,9 \\
\hline Age & & 1,9 E-09 & 0,000 & 1 \\
\hline Catégorie & & & & \\
Ouvriers & Employé & $-0,45$ & 0,04 & 0,63 \\
& Maîtrise & 0,60 & 0,03 & 1,82 \\
\hline Coefficient & & & & \\
antérieur & 215 & 1,78 & 0,02 & 5,8 \\
$155 / 190$ & 225 & 1,10 & N.S & 11,6 \\
& 240 & 2,33 & 0,001 & 34,9 \\
& 255 & 3,41 & 0,000 & 22,2 \\
& 270 & 3,20 & 0,000 & 69,2 \\
& 285 & 4,24 & 0,000 & 223,1 \\
\hline Diplôme & 305 & 5,45 & 0,000 & 1,8 \\
CAP & Bac & 0,58 & 0,01 & \\
& Bac+2 & $-0,23$ & N.S & N.S \\
\hline Autres & $-0,34$ & p.S & \\
\hline
\end{tabular}

Variable à tester: probabilité d'atteindre le coefficient 335 ou plus en 2003 pour les salariés qui sont devenus techniciens au cours de leur carrière. Variable discontinue $0=<305 ; 1=>335$

Individu de référence $=$ un homme, moins de 3 ans d'ancienneté, coefficient inférieur à 190, anciennement ouvrier, titulaire d'un CAP au moment de sa promotion.

Quand les promotions ont lieu, les Techniciens se situent majoritairement sur des coefficients assez faibles, près des $3 / 4$ (72\%) étaient en deçà du Niveau V (coefficient 305/335/365). Une fois promus, ils continuent de gravir des échelons. Cependant, leurs parcours n'est pas comparable à celui des Techniciens dans leur ensemble puisque les positions atteintes après le passage sont moins élevées. En 2003, $42 \%$ des promus sont au Niveau IV (30\% pour l'ensemble des Techniciens), $48 \%$ sont au Niveau V (61.5\% pour l'ensemble), et $4.5 \%$ au Niveau VI (6\%). Quant aux femmes, $60 \%$ d'entre elles ne dépassent pas le Niveau IV. Les chances des promus ne sont donc pas équivalentes à celle des autres Techniciens, les compteurs ne sont pas remis à zéro et tout se passe comme s'il n'était guère possible de cumuler deux carrières. L'expérience acquise précédemment représente plus un poids qu'un avantage. Pour les femmes, les handicaps se cumulent. Par rapport à l'ensemble des techniciennes, celles qui étaient auparavant principalement des employées vont donc se retrouver sur les plus bas échelons. Dans la hiérarchie des classements, en 2003, au sein de la population des Techniciens, on trouve donc au plus bas des grilles de classification, les femmes promues et anciennement employées, puis l'ensemble des femmes techniciennes, puis les hommes promus et anciennement ouvriers, et enfin les hommes techniciens. 
Tableau V

Accession aux différents Niveaux de classification en 2003

selon le type de population

\begin{tabular}{|c|c|c|c|c|}
\hline & $\begin{array}{c}\text { Niveau II/III } \\
190 / 215 / 225 / 240\end{array}$ & $\begin{array}{c}\text { Niveau IV } \\
255 / 270 / 285\end{array}$ & $\begin{array}{c}\text { Niveau V } \\
305 / 335 / 365\end{array}$ & $\begin{array}{c}\text { Niveau VI } \\
395 / 425\end{array}$ \\
\hline $\begin{array}{c}\text { F Promues } \\
\mathrm{N}=302\end{array}$ & $6 \%$ & $54 \%$ & $39 \%$ & $2 \%$ \\
\hline $\begin{array}{c}\text { Techniciennes } \\
\mathrm{N}=603\end{array}$ & $4 \%$ & $47 \%$ & $47 \%$ & $2 \%$ \\
\hline $\begin{array}{c}\text { H Promus } \\
\mathrm{N}=1124\end{array}$ & $4 \%$ & $39 \%$ & $51 \%$ & $5 \%$ \\
\hline $\begin{array}{c}\text { Techniciens } \\
\mathrm{N}=3330\end{array}$ & $2 \%$ & $27 \%$ & $64 \%$ & $6 \%$ \\
\hline
\end{tabular}

Lecture : sur 100 femmes devenant technicienne, 6 sont au Niveau II/III en 2003

\section{Devenir cadres}

Les mêmes observations s'imposent pour les Techniciens qui deviennent cadres. Par rapport à l'ensemble des cadres (2181) en 2003, 15\% (332) étaient des Techniciens au moment de leur entrée dans l'entreprise. Le profil général de ces promus ressemble par bien des aspects à celui des anciens ouvriers et employés devenus Techniciens. Dans la très grande majorité ce sont des hommes, ayant une moyenne d'âge de 35 ans et 12 ans d'ancienneté au moment du passage, ils sont principalement situés au Niveau IV (64\%) ${ }^{25}$. L'accession au statut de cadre se fait principalement dans la première partie de leur carrière $(72 \%$ ont moins de quinze ans d'ancienneté). Le passage cadre marque une rupture par rapport à la situation antérieure puisque le système de classification change ${ }^{26}$. Il n'existe pas de continuum entre les Niveaux et les Positions, ce sont deux systèmes parallèles, l'entrée dans l'univers des cadres suppose donc de repartir de zéro, alors que ce n'était pas le cas pour les ouvriers et les employés devenus Techniciens

Quelles sont leurs perspectives ? Leur parcours n'est pas semblable à celui des cadres dans leur ensemble, dont une très grande partie a eu accès au statut directement, sur la base du diplôme initial ${ }^{27}$. Les promus connaissent par la suite de la mobilité, cependant ils accèdent

\footnotetext{
25. Le profil des techniciens promus est très proche des candidats en formation continue souhaitant s'inscrire dans les «nouvelles filières d'ingénieurs » décrit par C. Gadéa, P. Loubet et P. Roquet, [C. Gadéa, P. Loubet et P. Roquet, 1996].

26. Pour la catégorie des cadres, on ne parle plus de coefficients, ni même de niveau. Les cadres se répartissent sur sept positions : I, II, III A, III B, III BS (Sup), III C, et DHS (Directeur hors statut).

27 En 2000, selon une enquête de l'APEC, 40\% des nouveaux entrants dans la catégorie des cadres sont des promus. [APEC, 2001]. Voire aussi, C. Gadéa, A. Rezrazi, [C. Gadéa, A. Rezrazi, 2004]. La part des promus au sein de l'entreprise paraît donc être très en deçà de ce qu'elle est ailleurs dans les grandes entreprises : en 2000, 114 cadres ont été recrutés, dont 24 sont des promus, soit $21 \%$.
} 
moins souvent que les autres cadres aux positions les plus hautes. Comme pour les ouvriers et employés promus Techniciens, l'hypothèse la plus plausible est de penser que la gestion de carrière des cadres qui entrent par la «petite porte » diffère de celle des cadres qui le sont depuis toujours. Ainsi, $90 \%$ des premiers sont situés sur les positions II et III A, alors que ce n'est vrai que pour $63 \%$ de l'ensemble des cadres en 2003. Pour les femmes, les perspectives sont encore plus restreintes. Sur l'ensemble des Techniciens qui deviennent cadres, les femmes sont sous-représentées. $12 \%$ sont promues alors que les femmes cadres comptent pour $17 \%$ de l'ensemble de ce groupe. Et sur l'ensemble des femmes cadres, $10 \%$ sont promues.

Comme pour les ouvrières et les employées devenues techniciennes, les critères pour accéder à la catégorie des cadres sont a priori peu discriminants. Elles deviennent cadres en moyenne en ayant une ancienneté dans l'entreprise un peu moindre que les hommes (61\% ont moins de dix ans d'ancienneté ; $35 \%$ pour les hommes), mais se situent sur les mêmes niveaux de classification que leurs collègues masculins. En revanche, une fois devenues cadre, elles accèdent moins souvent aux positions les plus hautes : $49 \%$ en Position II ; $43 \%$ pour les hommes, $3 \%$ en Position III B, $11 \%$ pour les hommes. Malgré la faiblesse des effectifs (39 femmes seulement), ces résultats dessinent bien une même tendance générale, à savoir que les femmes font l'objet d'un traitement particulier qui les pénalise quant aux perspectives de promotion ${ }^{28}$. La comparaison avec l'ensemble de la population cadre montre bien la nature de la hiérarchie. Les techniciennes qui deviennent cadre se retrouvent au plus bas du système de classement, puis viennent les femmes cadres dans leur ensemble, ensuite les techniciens ayant intégré l'univers des cadres et enfin l'ensemble des hommes cadres. Si l'on ne prend comme point de repère que l'accès à la position III $\mathrm{B}$, équivalant au statut de cadre supérieur, les femmes promues ont très peu de chance d'y accéder (3\%), et celles qui sont entrées dans l'entreprise avec ce statut se rapproche des hommes qui étaient auparavant techniciens $(12 \% ; 11 \%$ respectivement). À leur ancien statut vient s'ajouter leur domaine de spécialisation, les femmes en effet, qu'elles soient cadres à l'issue de leur formation, où le deviennent par le biais de la promotion, n’ont pas les « attributs du management généraliste et opérationnel », ceux qui ouvrent l'accès à la position de cadre supérieur. Elles ont plus souvent des profils de «spécialistes: gestions des ressources humaines, communication, marketing ou finance $»[$ J. Laufer, 2003].

28. Dans la régression logistique pour les Techniciens qui deviennent cadres, la variable sexe n'est pas significative. 
Tableau VI

Positions atteintes par les cadres sur les grilles de classification

\begin{tabular}{|c|c|c|c|c|c|c|c|}
\hline & en 2003 en fonction de leur parcours, \% \\
\hline $\begin{array}{c}\text { Promues } \\
\mathrm{N}=39\end{array}$ & 3 & 49 & 46 & 3 & 0 & 0 & 0 \\
\hline $\begin{array}{c}\text { F cadres } \\
\mathrm{N}=368\end{array}$ & 8 & 43 & 34 & 12 & 0,5 & 2 & 0 \\
\hline $\begin{array}{c}\text { Promus } \\
\mathrm{N}=293\end{array}$ & 0 & 43 & 45 & 11 & 1 & & \\
\hline $\begin{array}{c}\mathrm{H} \text { cadres } \\
\mathrm{N}=1813\end{array}$ & 5 & 28 & 32 & 20 & 5 & 8 & 2 \\
\hline
\end{tabular}

Lecture en ligne : sur 100 cadres promues, 3 sont situées sur la Position I en 2003.

Les femmes subissent un double stigmate quand elles appartiennent auparavant à une autre catégorie socio professionnelle: on peut distinguer les anciens techniciens qui s'apparentent à des «quasi-cadres », des techniciennes qui se rapprochent des «équivalentcadres » [C. Gadéa, P. Loubet, P. Roquet, 1996]. L'accès à la catégorie des cadres par les mécanismes de promotion est une filière qui résiste beaucoup plus aux femmes [C. Marry, 2004 ; J. Laufer, S. Pochic, 2004].

\section{Paralysie faCe AUX Discriminations}

Les bilans fournis par l'entreprise concernant la situation comparée des hommes et des femmes tendent à démontrer un traitement sinon égalitaire, du moins proche, et excluent ainsi toutes pratiques discriminatoires. Ce bilan n'est pas faux en soi, cependant, comme l'analyse des différentes situations l'a montré, il est trompeur et tronqué. Certes, l'entreprise ne fait que se conformer à la loi et utilise les outils réglementaires. Cependant, on peut faire l'hypothèse qu'en respectant scrupuleusement le cadre législatif l'entreprise masque une partie de la réalité par stratégie. Plusieurs éléments étayent cette thèse et soulignent les limites dans lesquelles les accords sur l'égalité hommes femmes se construisent.

Le rapport, joint au bilan social, est destiné aux partenaires sociaux. L'égalité hommes femmes est un sujet relativement consensuel et l'entreprise n'a évidemment pas intérêt à fournir un document présentant un bilan sinon négatif, tout du moins imparfait, et laissant apparaître de nombreuses zones d'ombre. Par ailleurs, les accords sont renégociables au bout de trois ans. Or, les relations avec les syndicats s'avèrent plus ou moins tendues du fait de l'accumulation de décisions stratégiques de l'entreprise sur lesquelles les partenaires sociaux n'ont aucune prise. Il en va ainsi du recours croissant à la sous-traitance, d'une délocalisation 
de certaines activités vers la Chine et l'Inde, de l'absence d'embauche depuis plusieurs années et du non-renouvellement des départs à la retraite, et enfin d'une ouverture du capital puis d'une privatisation partielle ${ }^{29}$. Autant d'éléments sur lesquels les syndicats manifestent leurs désapprobations mais contre lesquels ils n'ont guère d'emprise. Dans ce contexte, intégrer dans le rapport sur l'égalité hommes femmes des indicateurs tenant compte du parcours des salariés, indicateurs à la seule disposition du service du personnel, c'est du point de vue de l'entreprise prendre le risque d'offrir aux syndicats des leviers de contestation d'autant plus grands qu'ils sont légitimes au regard de la loi et auprès des salariés puisque le sujet est consensuel.

Prendre en compte le parcours des salariés c'est donc exposer les différences de parcours et d'opportunités entre les hommes et les femmes et le décrochage qu'elles subissent tout au long de leur carrière. C'est en réalité pour l'entreprise reconnaître des formes de discrimination à l'égard des femmes. Or la notion de discrimination est ambivalente auprès des acteurs car elle est spontanément associée à l'idée de pratiques volontaires, à un mode délibéré de gestion différenciée pour les hommes et pour les femmes. La discrimination prend alors une dimension restrictive qui voudrait qu'elle n'existe que si elle résulte d'une politique volontaire et explicite. Ce faisant, les acteurs en charge de ce dossier au sein du service du personnel tendent à la sous estimer car ils ne perçoivent pas que la discrimination procède d'une multitude de pratiques, que les acteurs peuvent légitimer et rationaliser localement, et qui mises bout à bout engendrent des effets non voulus. C'est ce que montre J. Laufer à propos des femmes cadres à haut potentiel qui ne parviennent que très rarement aux postes de direction car elles sont pénalisées non pas aux vues de leur compétence réelles mais aux vues des compétences présupposées pour accéder à ces niveaux puisqu'elles ont plus rarement eu des postes de management généraliste et opérationnel. «Les normes culturelles et managériales forgent explicitement mais aussi implicitement les modèles du manager idéal, or ces modèles semblent plus facilement se décliner au masculin qu'au féminin » [J. Laufer, 2003]. Autant de représentations qui se jouent bien en amont, dans les choix et les conseils d'orientation scolaire. Dans ce cas aussi, penser, par exemple, que les filles ont moins d'aptitude ou de goût pour la technique, c'est les exclure de ce domaine sans avoir eut l'intention de le faire [M. Duru-Bellat, 2002]. Pour l'entreprise, la discrimination relève d'une

29. L'entreprise à été nationalisée après la seconde guerre mondiale. Dans les années 1970, l'état cède environ $10 \%$ du capital à un grand groupe industriel américain, partenaire privilégié de l'entreprise. Dans les années 1990 l'ouverture du capital est envisagée. Elle aurait dû avoir lieu en 2001, les événements du 11 septembre ont arrêté le processus. En 2004, l'ouverture du capital s'effectue puis, six mois après, à la suite d'une fusion avec un groupe français, l'état ne détient plus que $30 \%$ du capital. 
politique, à ce titre elle n'existe pas, alors que bien plus souvent elle résulte de pratiques discriminantes [Ph. Bataille, 1997]. Ainsi, ne percevoir les inégalités que sous l'angle d'une politique délibérée conduit à occulter le phénomène et à le reproduire.

Reconnaître la discrimination, directe ou indirecte, soulève pour l'entreprise l'épineuse question de l'exposition au cadre de la loi et donc à des sanctions. En effet, la direction du personnel est prise entre deux logiques contradictoires. Elle cherche à promouvoir l'égalité des chances et à ce titre, par exemple, elle inclut dorénavant les femmes en congé de maternité dans les effectifs. Ce qui signifie concrètement qu'elles peuvent bénéficier des augmentations individuelles, voire d'une promotion, ce qui n'était pas le cas auparavant puisqu'elles étaient momentanément sorties des effectifs. Cependant, si la direction du personnel tente de garantir l'égalité de traitement, elle doit donc reconnaître préalablement les écarts et s'exposer ainsi à des plaintes et à des procès. Or, comme la loi sur l'égalité professionnelle intègre les directives de la cour de justice européenne, en particulier le renversement de la preuve, c'est dorénavant à l'entreprise de justifier la politique poursuivie [M-T. Lanquetin, 1998]. Stratégiquement, si la commission égalité professionnelle livre un état de la situation, elle a aussi comme premier souci de parer aux attaques et de protéger l'entreprise contre les plaintes. Elle ne peut se permettre de livrer un document qui se retournerait contre elle. Formellement, l'entreprise et la direction du personnelle ne disposent pas des données présentées et analysées ci dessus. Toutefois, elles ne cherchent pas et ne souhaitent pas y avoir accès. Ainsi, dans le cadre de l'enquête menée dans l'entreprise, les acteurs en charge du dossier n'ont pas voulu les exploiter lorsque je leur ai présenté les tableaux, comme ils m'ont demandé de substituer systématiquement, dans le rapport que je leur ai remis, le terme de discrimination par celui d'inégalité. Malgré ces changements, le rapport a été jugé non diffusable.

Ce souci d'occulter le problème est d'autant plus grand et présent que l'entreprise est confrontée par ailleurs à un dossier de discrimination syndicale et qu'elle négocie avec la principale organisation pour éviter que le dossier se traite aux prud'hommes. À ce propos, on parle de compensation s'élevant à plusieurs millions d'Euros. En période d'ouverture du capital, l'entreprise ne peut se permettre de voir son image se ternir et devenir la cible des médias.

Ainsi, il n'y a pas de volonté de discrimination. Par contre stratégiquement l'entreprise prend moins de risque à gommer les écarts entre les hommes et les femmes, en s'en tenant strictement au cadre de la loi, tout en affichant sa volonté de promouvoir l'égalité des chances, qu'à aborder la question de front et de donner ainsi des armes aux syndicats. S'il est 
possible d'évoquer en privé cette question, elle relève du tabou dans le cadre de la politique de l'entreprise parce que le sujet est étroitement associé à la perception d'un retournement contre l'entreprise. Parler de discrimination suscite la crainte et incite à des stratégies défensives et de repli. «On ne parle pas de discrimination, car ce n'est pas une politique délibérée, comme il y a eu avec les syndicats. Puis, avec le terme de discrimination, il y a le risque de contentieux », explique une responsable de la commission. Parler de discrimination, c'est reconnaître une «faute». "Quand on est une femme, on sait qu'il ne faut pas dévier. Ceux avec qui je travaillais, ont tous eu des promotions, sauf moi. J'ai eu une mobilité horizontale, pas verticale. Mais je n'ai jamais de remarque sur le travail. On ne peut pas exprimer ces problèmes, surtout quand on est au service du personnel. On ne peut pas en parler car il y a une volonté au niveau société de ne pas faire de discrimination. Si on l'exprime, je risque de perdre plus de plumes que d'en gagner. C'est d'autant plus dur que c'est peu factuel, car il y a peu de femmes, donc statistiquement c'est difficile de le montrer. On rétorquera toujours que les effectifs sont trop faibles pour que les données soient significatives. Donc j'accepte d'être politiquement correct sur ce problème et de ne rien dire. J'en suis à me dire que de toute manière, je n'ai pas forcément les éléments nécessaires pour obtenir plus, donc à intérioriser et à anticiper le problème. »

Au-delà de l'aspect stratégique conduisant à éviter d'aborder de front la question des discriminations, un des premiers obstacles à leur prise en charge tient à leur absence de visibilités. Pour que la discrimination à l'égard des femmes devienne un enjeu, encore faut-il que le phénomène soit perçu et nommé en tant que tel, qu'il passe du statut de faits-divers à celui de problème social. Or, aux dires de différents acteurs, et malgré l'accord signé entre la Direction Générale et les cinq organisations syndicales, les pratiques discriminatoires restent peu visibles du fait même de la marginalisation des femmes au sein de l'entreprise. Aucune, par exemple, n'a le grade de Directeur hors Statut dans les effectifs de 2003. Comme le suggère $\mathrm{J}$ Laufer, la présence minoritaire des femmes dans le cercle des dirigeants « est de toute évidence l'une des dimensions qui conduit à la persistance du plafond de verre ». Aborder la question des inégalités hommes/femmes dans les stratégies de l'entreprise et dans les perspectives des gestions de carrière nécessite donc des efforts et une volonté particulière car elles sont pratiquement absentes des instances où se décident les grandes orientations. «Pour nous, estime une responsable impliquée dans ce dossier, il n'y a pas de discrimination volontaire, c'est plus une question de mentalité. Il n'y a pas de consigne officielle. Mais les directions ont fermé les yeux sur un phénomène. Quand des choix sont faits, il n'y a aucune femme autour de la table. Donc la question du choix homme/femme ne se pose pas ». 
L'invisibilité immédiate et l'embarras que suscite ce thème conduit les acteurs à des formes de contorsion complexes, où il s'agit d'évoquer le problème sans vraiment le mentionner, de l'aborder sans pour autant lui accorder trop de place et d'importance. Ce thème met mal à l'aise, parce qu'il génère des formes de culpabilité qui sont d'autant plus grande qu'individuellement les acteurs n'en sont pas directement responsables. Le paradoxe de cette situation est que personne ne nie formellement que les inégalités existent, puisque pour les justifier chacun évoque notamment le passé de l'entreprise où les femmes étaient marginales et essentiellement cantonnées dans les activités tertiaires ou à la direction de la production dans des niches très spécifiques. Faire référence au passé permet de justifier un phénomène et joue comme une forme de déculpabilisation, comme si le problème était essentiellement perçu comme étranger à l'entreprise. Il a existé et tout le monde feint de croire qu'il n'existe plus : la preuve, le rapport souligne les faibles écarts entre les hommes et les femmes sur les indicateurs retenus. Faire état du problème et le reconnaître publiquement s'apparente, au contraire, à une mise en cause de la gestion des carrières et revient à rendre visible ce qui ne doit pas l'être et donc à s'exposer à des plaintes. «Parler de discrimination, c'est soulevé le côté négatif, explique une responsable de la commission. Or, ici c'est : il n'y a pas de problèmes, il y a des actions d'amélioration. Ça veut dire qu'inconsciemment on n'a pas fait le pas. On préfère ne pas l'aborder comme tel ».

L'exploration du parcours des Techniciens et la mise en évidence des inégalités des opportunités de carrière pour les femmes, ne saurait remettre en cause les lois sur l'égalité professionnelle et les outils à la disposition des entreprises et des acteurs. Elle montre en revanche plus généralement, et plus simplement aussi, la multiplicité des critères et des formes des inégalités [F. Dubet, 2000]. L'égalité formelle telle qu'elle apparaît dans les bilans de l'entreprise n'est pas incompatible, et s'accommode fort bien, avec d'autres formes d'inégalités, plus structurelles. Les inégalités ne forment pas un système, elles ne se présentent pas comme un bloc compact uniforme. Au contraire, selon les critères retenus, et les points de vue adoptés, elles divergent, peuvent s'atténuer, se stabiliser ou se creuser. La question qui se pose alors pour les entreprises et les acteurs directement engagée dans cette problématique, à travers la question des outils, et de savoir à quel niveau il est opportun et nécessaire d'agir. Dit autrement, et en reprenant le vocabulaire de la philosophie politique, 
quelles sont les inégalités justes, acceptables, que l'entreprise, les partenaires sociaux et ici les femmes, peuvent tolérer ou supporter?

Or ce travail, complexe et délicat, n'est que rarement mené puisque les inégalités sont abordées comme un tout. Tout le monde s'accorde sur la nécessaire lutte contre les inégalités et refuse toute forme de discrimination. Mais chacun parle-t-il de la même chose ? Afficher une égalité formelle des salaires entre les hommes et les femmes à coefficient équivalent, mais s'accommoder des inégalités dues aux différences de parcours et de filières est-ce plus acceptable que d'introduire des mesures compensatoires visant à rattraper les inégalités structurelles au risque de générer des inégalités par la création de populations prioritaires ? Bref, l'égalité de traitement sur la base d'une inégalité des conditions est-elle préférable à ce qu'on nomme plus communément la discrimination positive qui, elle, introduit au contraire de l'inégalité pour tenter d'y remédier? Force est de constater que ce débat suscite des prises de positions parfois très tranchées au sein de la société française. Il peine à être véritablement posé dans le cadre de l'institution scolaire, qui est pourtant une des première à l'avoir introduit, et reste toujours sous la menace de directives contradictoires au gré des changements ministériels et des pressions syndicales. Il est encore marginal et souterrain dans les entreprises, probablement parce que le dialogue social reste à conquérir et parce qu'elles sont plus soucieuses d'éviter les sanctions pénales et les contentieux, au risque de se cacher une partie de leur pratique. 


\section{Bibliographie}

Bataille. Ph, 1997, Le racisme au travail, Paris, La Découverte.

Bouffartigue. P, Gadéa. C, 1996, «Un héritage à l'épreuve. Bref panorama des évolutions dans la formation et l'emploi des ingénieurs en France », Formation Emploi, n 53 , p. 5-13.

Bourdieu. P, 1991, La noblesse d'Etat. Grandes écoles et esprit de corps, Paris, Ed. de Minuit.

Daune-Richard. A-M, 1998, "Qualification et représentation sociale », in M. Maruani, Femmes, genre et société, l'état des savoirs, Paris, La Découverte.

Dauverchain. A, 2003, L'égalité homme femme au travail, DESS, Université de Lyon II.

Dubet. F, 2000, Les inégalités multipliées, La Tour d'Aigues, éd. de l'Aube.

Dupray. A, Moullet, S, 2005, «Les salaires des hommes et des femmes. Des progressions particulièrement inégales en début de vie active », CEREQ, Bref, n²19, mai.

Duru-Bellat. M, 1990, L'école des filles, Paris, L'Harmattan.

Duru-Bellat. M, 2002, Les inégalités sociales à l'école, Paris, PUF.

Fakhfkh. F, Lemière. S, Merlateau, M-P, Meurs. D, 2004, «Les disparités de rémunération entre hommes et femmes: la situation de quatre branches professionnelles », DARES, Document d'études, $\mathrm{n}^{\circ} 80$, janvier.

Fortino. S, 2004, «Rapport sociaux de sexe et classe sociale », in P. Bouffartigue (dir.), Le retour des classes sociales, Paris, La Dispute.

Gadéa. C, Loubet. P, Roquet. P, 1996, «Ingénieurs en puissance. Formation professionnelle des techniciens et ordre négocié de la sélection », Formation Emploi, n55, p. 43-57.

Gadéa. C, Rezrazi. A, 2004, «Promotion et genre : une mosaïque de différences », in A. Karvar, L. Rouban (ss. dir.), Les cadres au travail, Paris, la Découverte.

Joseph. O, Lemière. S, 2005, « La discrimination de genre et d'origine à l'encontre des jeunes sur le marché du travail », CEREQ, Net.Doc.12, mars.

Lanquetin. M-T, 1998, «L'égalité professionnelle : le droit à l'épreuve des faits », in M. Maruani (dir), Femmes, genre et société, l'état des savoirs, Paris, La Découverte.

Laufer. J, 2003, «L'accès des femmes à la sphère de direction des entreprises: la construction du plafond de verre », DARES, rapport de synthèse, octobre.

Laufer. J, 2005, «L'égalité professionnelle », in M. Maruani (dir), Femmes, genre et société, l'état des savoirs, Paris, La Découverte.

Laufer. J, S. Pochic, 2004, «carrière au féminin et au masculin », in A. Karvar, L. Rouban (dir.), Les cadres au travail, Paris, La Découverte.

Le Minez. S, Roux. S, 2001, «Les écarts de revenu salarial entre hommes et femmes en début de carrière », INSEE Première, $\mathrm{n}^{\circ}$ 801, août.

Marry. C, 1997, Le diplôme et la carrière : masculin/féminin, in J-P. Terrail (dir.), La scolarisation en France, Paris, la Dispute.

Marry. C, 2004, Les femmes ingénieurs. Une révolution respectueuse, Paris, Belin

Silvera. R, 1998, «Les salaires : toutes choses inégales par ailleurs ?», in M. Maruani (dir.), Les nouvelles frontières de l'inégalité, Paris, La Découverte ; INSEE, DADS 2003

Stiglitz. J-E, 1973, «Approaches to the Economics of Discrimination », The American Economic Review, vol 63, n², May, p. 287-295.

Vallet. L-A, Caille. J-P, 1996, « Les élèves étrangers ou issus de l'immigration dans l'école et le collège français », Les dossiers d'Education et Formations, n ${ }^{\circ} 67$.

Veneau. P, 1995, «Evolution de la mobilité et accès à la position de technicien », Sociétés contemporaines, $\mathrm{n}^{\circ} 22$, p. 185-206. 


\begin{abstract}
ANNEXE
Les données exploitées dans cet article proviennent d'une enquête sociologique menée pendant un an et demi dans une grande entreprise du secteur de la métallurgie. Lors de mon séjour dans cette entreprise, j'ai été conduit à produire un rapport sur la population des techniciens. J'ai donc pu avoir accès à certains fichiers du personnel dans lesquels sont consignées plusieurs informations. Sur la base de ces fichiers, j'ai pu en élaborer d'autres, pour reconstruire notamment les cohortes et les parcours des promus. Il n'a pas été possible de remonter plus loin que l'année 1995 et les informations ne sont pas toujours comparables car les nomenclatures ont souvent changées plusieurs fois tout au long de cette période.
\end{abstract}

1). Indicateurs, définis par l'article D. 432-1 du code du travail, devant figurer dans le rapport annuel relatif à la situation comparée des hommes et des femmes.

Effectifs : données chiffrées par sexe : répartition par catégorie professionnelle selon les différents contrats de travail. Pyramide des âges par catégorie professionnelle.

Durée et organisation du travail : données chiffrées par sexe : répartition des effectifs selon la durée du travail. Répartition selon l'organisation du travail.

Données sur les congés : données chiffrées par sexe : répartition par catégorie professionnelle selon le nombre et le type de congé dont la durée est supérieur à 6 mois.

Données sur les embauches et les départs : données chiffrées par sexe : répartitions des embauches par catégorie professionnelle et type de contrat de travail. Répartition des départs par catégorie professionnelle et motifs.

Positionnement dans l'entreprise: données chiffrées par sexe: répartition des effectifs selon les niveaux d'emplois définis par les grilles de classification au sens des conventions collectives.

Promotions : données chiffrées par sexe : répartition par promotion au regard des effectifs et de la catégorie professionnelle concernée. Nombre de promotions suite à une formation.

Rémunérations: données chiffrées par sexe: éventail des rémunérations. Rémunération moyenne mensuelle. Nombre de femmes dans les 10 plus hautes rémunérations.

Formation: données chiffrées par sexe et par catégorie professionnelle : participation aux actions de formation. La répartition par type d'action. Le nombre moyen d'heure d'action de formation.

Conditions de travail : données chiffrées par sexe et répartition selon le poste de travail : l'exposition à des risques professionnels. La pénibilité, dont le caractère répétitif des tâches.

2) Régression logistique pour les techniciens de 1995, interaction sexe/ancienneté

Tableau VIII

Chances d'accéder aux coefficients les plus élevés pour les Techniciens en 1995

Régression logistique

\begin{tabular}{|c|l|c|c|c|}
\hline Variable & \multicolumn{1}{|c|}{ Modalité } & B & Sig & Odds Ratio \\
\hline Constante & & $-6,95$ & 0,000 & \\
\hline Sexe/ancienneté & & & & \\
Hommes débutant & Hommes 11/19 ans & 1,37 & 0,000 & 3,94 \\
& Hommes 20/41 ans & 1,84 & 0,000 & 6,34 \\
& Femmes débutantes & $-0,42$ & N.S & \\
& Femmes 11/19 ans & $-0,06$ & N.S & \\
& Femmes 20/41 ans & 0,67 & 0,02 & 1,9 \\
\hline Age & & 0,14 & 0,000 & 1,15 \\
\hline \multirow{2}{*}{ Diplôme } & & & & \\
Bac +4 & CAP & $-2,06$ & 0,000 & 0,12 \\
& Bac & $-0,96$ & 0,000 & 0,38 \\
& Bac+2 & 0,45 & N.S & \\
\hline Directions & & & & \\
Technique & Autres & $-0,24$ & N.S & \\
& Civile & $-0,52$ & 0,02 & 0,59 \\
& Militaire & 0,15 & N.S & \\
& Production & $-0,51$ & 0,00 & 0,59 \\
& Qualité & $-0,30$ & 0,01 & 0,74 \\
\hline
\end{tabular}

Variable à tester : probabilité d'atteindre le coefficient 335 ou plus pour les techniciens. Variable discontinue $0=<$ $335 ; 1=>335$

Lecture : L'individu de référence est un homme ayant entre 3 et 10 ans d'ancienneté, bac+4, direction technique. 
3) Régression logistique pour les techniciens en 2003

Tableau VII

Chances d'accéder aux coefficients les plus élevés pour les Techniciens en 2003

\begin{tabular}{|l|l|c|c|c|}
\multicolumn{5}{c|}{ Régression logistique } \\
\hline Variable & Modalité & B & Sig & Odds Ratio \\
\hline Constante & & $-8,32$ & 0,000 & \\
\hline $\begin{array}{c}\text { Sexe } \\
\text { Femme }\end{array}$ & Homme & 0,95 & 0,000 & 2,59 \\
\hline Ancienneté & & 0,09 & 0,000 & 1,1 \\
\hline Age & & 0,1 & 0,000 & 1,1 \\
\hline Diplôme & & & & \\
Bac +4 & CAP & $-1,9$ & 0,000 & 0,14 \\
& Bac & $-0,81$ & 0,017 & 0,44 \\
& Bac+2 & 0,56 & N.S & \\
\hline Directions & & & & \\
Technique & Autres & $-0,14$ & N.S & \\
& Qualité & $-0,23$ & N.S & \\
& Production & $-0,61$ & 0,000 & 0,54 \\
& Civile & $-1,54$ & 0,000 & 0,21 \\
& Militaire & 0,2 & N.S & \\
\end{tabular}

Variable à tester : probabilité d'atteindre le coefficient 365 ou plus pour les techniciens. Variable discontinue $0=<$ $335 ; 1=>365$

Lecture : L'individu de référence est une femme, avec un an d'ancienneté, ayant un bac+4, travaillant à la direction technique. Les hommes ont 2,6 fois plus de chances d'être au coefficient 365 et plus que les femmes.

4) Régression logistique pour les techniciens de 2003, interaction sexe/ancienneté

Tableau IX

Chances d'accéder aux coefficients les plus élevés pour les Techniciens en 2003

Régression logistique

\begin{tabular}{|l|l|c|c|c|}
\hline Variable & \multicolumn{1}{|c|}{ Modalité } & B & Sig & Odds Ratio \\
\hline Constante & & $-6,85$ & 0,000 & \\
\hline Sexe/ancienneté & & & & \\
Hommes débutant & Hommes 20/24 ans & 0,98 & 0,000 & 2,68 \\
& Hommes 25/41 ans & 1,63 & 0,000 & 5,13 \\
& Femmes débutantes & $-0,87$ & N.S & \\
& Femmes 20/24 ans & 0,06 & N.S & 1,9 \\
\hline Age & Femmes 25/41 ans & 0,64 & 0,03 & 1,12 \\
\hline \multirow{2}{*}{ Diplôme } & & 0,11 & 0,000 & \\
Bac +4 & CAP & $-1,8$ & 0,000 & 0,16 \\
& Bac & $-0,79$ & 0,01 & 0,44 \\
Directions & Bac+2 & 0,58 & N.S & \\
Technique & Autres & $-0,18$ & N.S & \\
& Qualité & $-0,25$ & N.S & \\
& Production & 0,61 & 0,00 & 0,53 \\
& Civile & $-1,56$ & 0,00 & 0,2 \\
& Militaire & 0,21 & N.S & \\
\hline
\end{tabular}

Variable à tester : probabilité d'atteindre le coefficient 365 ou plus pour les techniciens. Variable discontinue $0=<$ $335 ; 1=>365$

Lecture : L'individu de référence est un homme ayant entre 1 et 20 ans d'ancienneté, ayant un bac+4, travaillant à la direction technique. 
5). Table de mobilité pour les Techniciens 2003/1995.

Tableau X

Table de mobilité : positions atteintes en 2003 par les techniciens présents en 1995 en fonction des coefficients où ils étaient situés, \%

\begin{tabular}{|l|c|c|c|c|c|c|c|c|c|c|}
\hline & $<240$ & 255 & 270 & 285 & 305 & 335 & 365 & $>395$ & Total & T \% \\
\hline$<240$ & 15 & 31 & 35 & 16 & 3 & & & & 170 & 7 \\
\hline 255 & & 4 & 35 & 49 & 11 & 1 & & & 178 & 8 \\
\hline 270 & & & 7 & 40 & 48 & 4 & 1 & & 333 & 15 \\
\hline 285 & & & & 12 & 58 & 30 & 1 & & 477 & 21 \\
\hline 305 & & & & & 17 & 67 & 16 & & 554 & 25 \\
\hline 335 & & & & & & 31 & 63 & 6 & 382 & 17 \\
\hline 365 & & & & & & & 45 & 55 & 121 & 5 \\
\hline$>395$ & & & & & & & & 100 & 37 & 2 \\
\hline Total & 26 & 59 & 146 & 304 & 553 & 647 & 388 & 129 & 2252 & \\
\hline$\%$ T & 1 & 3 & 6 & 13 & 25 & 29 & 17 & 6 & & \\
\hline
\end{tabular}

Lecture en ligne : Sur 100 techniciens situés sur un coefficient inférieur à 240 (Niveaux II et III) en 1995, 15 sont toujours au même niveau, 31 sont désormais au coefficient 255 en 2003. Les coefficients supérieurs à 395 correspondent au Niveau VI.

Tableau XI

Table de mobilité : positions atteintes en 2003 par les techniciennes présents en 1995 en fonction des coefficients où ils étaient situés, \%

\begin{tabular}{|l|c|c|c|c|c|c|c|c|c|c|}
\hline & $<240$ & 255 & 270 & 285 & 305 & 335 & 365 & $>395$ & Total & $\mathrm{T} \%$ \\
\hline$<240$ & 17 & 42 & 33 & 8 & & & & & 76 & 20 \\
\hline 255 & & 6 & 40 & 41 & 12 & 0 & & & 82 & 22 \\
\hline 270 & & & 13 & 48 & 35 & 4 & & & 71 & 19 \\
\hline 285 & & & & 19 & 49 & 32 & & & 47 & 12 \\
\hline 305 & & & & & 22 & 68 & 9 & & 63 & 17 \\
\hline 335 & & & & & & 29 & 67 & 4 & 24 & 6 \\
\hline 365 & & & & & & & 67 & 33 & 12 & 3 \\
\hline$>395$ & & & & & & & & 100 & 3 & 3 \\
\hline Total & 13 & 37 & 67 & 83 & 72 & 68 & 30 & 8 & 378 & \\
\hline$\%$ T & 3 & 10 & 18 & 22 & 19 & 18 & 8 & 2 & & \\
\hline
\end{tabular}

Lecture en ligne : Sur 100 techniciennes situées sur un coefficient inférieur à 240 (Niveaux II et III) en 1995, 17 sont toujours au même niveau, 42 sont désormais au coefficient 255 en 2003. 
6) Régression logistique pour les techniciens de 2003, interaction sexe/ancienneté et type de métier

Tableau XII

Chances d'accéder aux coefficients les plus élevés pour les Techniciens en 2003

Régression logistique

\begin{tabular}{|c|c|c|c|c|}
\hline Variable & Modalité & B & Sig & Odds Ratio \\
\hline Constante & & $-7,31$ & 0,000 & \\
\hline $\begin{array}{l}\text { Sexe/ancienneté } \\
\text { Hommes débutant }\end{array}$ & $\begin{array}{l}\text { Hommes } 20 / 24 \text { ans } \\
\text { Hommes } 25 / 41 \text { ans } \\
\text { Femmes débutantes } \\
\text { Femmes } 20 / 24 \text { ans } \\
\text { Femmes } 25 / 41 \text { ans }\end{array}$ & $\begin{array}{l}0,99 \\
1,63 \\
-0,71 \\
0,22 \\
0,82 \\
\end{array}$ & $\begin{array}{c}0,000 \\
0,000 \\
\text { N.S } \\
\text { N.S } \\
0,009 \\
\end{array}$ & $\begin{array}{l}2,69 \\
5,14 \\
\\
2,28\end{array}$ \\
\hline Age & & 0,12 & 0,000 & 1,12 \\
\hline $\begin{array}{l}\text { Diplôme } \\
\mathrm{Bac}+4\end{array}$ & $\begin{array}{l}\text { CAP } \\
\mathrm{Bac} \\
\mathrm{Bac}+2\end{array}$ & $\begin{array}{c}-1,8 \\
-0,86 \\
0,51\end{array}$ & $\begin{array}{c}0,000 \\
0,012 \\
\text { N.S }\end{array}$ & $\begin{array}{l}0,15 \\
0,42\end{array}$ \\
\hline $\begin{array}{l}\text { Directions } \\
\text { Technique }\end{array}$ & $\begin{array}{l}\text { Autres } \\
\text { Qualité } \\
\text { Production } \\
\text { Civile } \\
\text { Militaire } \\
\end{array}$ & $\begin{array}{c}-0,07 \\
-0,29 \\
-0,56 \\
-1,53 \\
0,3\end{array}$ & $\begin{array}{l}\text { N.S } \\
\text { N.S } \\
0,00 \\
0,00 \\
\text { N.S }\end{array}$ & $\begin{array}{l}0,56 \\
0,21\end{array}$ \\
\hline $\begin{array}{l}\text { Métiers } \\
\text { "féminin " }\end{array}$ & "Non féminin" & 0,44 & 0,002 & 1,56 \\
\hline
\end{tabular}

Variable à tester : probabilité d'atteindre le coefficient 365 ou plus pour les techniciens. Variable discontinue $0=<$ $335 ; 1=>365$

Lecture : L'individu de référence est un homme ayant entre 1 et 20 ans d'ancienneté, ayant un bac+4, travaillant à la direction technique et exerçant un métier « féminin ». 